\title{
Editors' Note
}

\author{
David C. Hofmann, Carlie L. Leroux-Demir, and Noorin Manji \\ University of Waterloo, Department of Sociology and Legal Studies
}

Since the publication of our first issue in October 2012, we received an overwhelming response to our last call for papers and saw immense growth in our readership. With these changes, we collectively decided to expand the CGJSC editorial team and hire a new group of talented and passionate staff members who have already contributed greatly to the journal. We would like to welcome to CGJSC our new assistant editors: Nick Athey, Erin Denton, Christina DeRoche, Julie Hagan, and Dikla Yogev; and, our new copyeditors: Jason Huang, Asheka Jackson, Eshan Jozaghi, Colin Scott, and Kim Seida! In addition to a growth in staff, CGJSC's website has also undergone some changes as the journal's readership continues to change and grow. Our new front-page website can be found at www.cgjsc.ca. Here you will find our updated masthead with information on our new staff, links to previous issues, updated media and photos from CGJSC events, and links to CGJSC's Online Journal System (OJS) site where authors can continue to submit their works. We are also pleased to announce that CGJSC will be partnering with the Canadian Network for Research on Terrorism, Security and Society (TSAS), for a special issue to be published in Fall 2013. Dr. Aurelie Campana, the Canada Research Chair on Terrorism and Conflicts has agreed to serve as guest editor and TSAS has generously donated $\$ 500$ to all the authors of accepted papers for this issue.

Once a year, we would like to take the time to sincerely thank our peer reviewers for their hard work and dedication. We would encourage you to consult the journal's masthead for a full list of all our reviewers who have helped over the last two issues (see: http://www.cgjsc.ca/masthead/peer-reviewers).

For our second issue, we have four highly interesting articles. Maureen Kihika's work, entitled "Ghosts and Shadows: A History of Racism in Canada" eloquently crafts the argument that the effects of institutionalized racism that has spanned several centuries, can still be felt by Afro-Canadians participating in the labour market today. Following Bakan's assertion that racist and hegemonic practices are heavily embedded in Canada's political structure, Kihika chronologically traces the consequences of neo-liberal policies, asserting that these policies, on the surface, appear to support the ideals of multiculturalism and inclusivity, but in reality, have only served to further propagate the capitalist and imperialist agendas of those in power. As a result, it is evidenced through official government statistics and past research, that Afro-Canadians continue to struggle economically, and are positioned within the lower rung of the, as Kihika notes, labor market hierarchy. With little earning power and few opportunities for stable employment, the author suggests that this particular racialized group continues to suffer the illconsequences of age old structures of systemic oppression.

Nicole Etherington's piece, "Educational Trajectories and Health over the Life Course: A Role for the DSBN Academy?," focuses on the noted link between socioeconomic stratification and health inequality by specifically examining the implementation of the District School Board of Niagara (DSBN) Academy in the Niagara region of Ontario, Canada. Research has shown that though, on average, one in three people over the age of twenty-four in Ontario have a university education, within the Niagara region, the rate of post-secondary education is far lower, at just one in five people. The District School Board of Niagara created the DSBN Academy in 2011 in response to these low rates with the awareness that individuals who do not attend or graduate successfully from a post-secondary institution are more likely to experience both poor physical and mental health later in life. Etherington discusses the intended goals of the DSBN Academy to offer social and academic programs designed to encourage students to not only graduate high school, but also successfully pursue a post-secondary education as well. With an in-depth discussion of the cumulative advantages and disadvantages that occur in the realms of education and health, the author evaluates the impact of the DSBN Academy and concludes that the Academy has the potential to offer students resources to attain additional forms of capital as a way to counteract the impacts of initial capital on educational success, and therefore health outcomes later in life. 
Konstantin Petoukhov's work, "Transforming the Legacy of Indian Residential Schools in Canada into a Public Issue: A Critical Analysis of Michael Burawoy's Public Sociology," interestingly uses Michael Burawoy's four types of sociology to frame the transformative process of 'private troubles' into "public issues' within the context of Indian Residential Schools (IRS). Petoukhov begins by providing an initial discussion of the background and outcomes of IRS that have long reverberated in indigenous communities, though IRSs were all officially closed by 1996. Petoukhov focuses her analysis on evaluating Burawoy's four types of sociology- policy, critical, professional, and public - on their ability to identify the private troubles that have arisen from residential school experiences into issues of public concern and systemic origins. The author, in detail, explores each of Burawoy's four forms and ultimately concludes that they are inconsistent in their capacity to contribute to an understanding of the private troubles created by IRS as public issues.

Ehsan Jozaghi's piece, “" 'The biggest mistake God ever made was to create junkies': Unsafe injection practices, health care discrimination and overdose deaths in Montreal, Canada" is centered on the issue of shared drug equipment by injection drug users (IDUs) in Montreal, Canada that has lead to an increased prevalence of HIV and Hepatitis C (HCV) since 2003. Jozaghi's research is based on qualitative narratives produced through semi-structured interviews with IDUs that revealed their involvement in highrisk behaviors that increase exposure to $\mathrm{HIV}, \mathrm{HCV}$, and bacterial transmission for both drug users and for the general public. The author discusses that some of the cumulative factors contributing to the increased morbidity and mortality of Montreal IDUs, as well as risk for the general public include: using contaminated puddle water for injection, being force to inject in unsanitary environments like public washrooms or alleys, discarding used needles in public spaces, and prejudice and harassment by police and hospital staff when seeking treatment. Jozaghi concludes with a recommendation that Montreal would greatly benefit from implementing a supervised injection facility, similar to other Canadian cities such as Vancouver.

Again we would like to extend a thank you to everyone who has contributed to the success of CGJSC, and to our readers and followers that have lent their support through readership and social networks. On behalf of CGJSC, please enjoy this exciting second issue! 


\title{
Note des rédacteur(ice)s
}

\author{
David C. Hofmann, Carlie L. Leroux-Demir, and Noorin Manji \\ University of Waterloo, Department of Sociology and Legal Studies
}

Depuis la publication de notre premier numéro en Octobre 2012, notre dernier appel à communications a eu un retentissement exceptionnel et nous avons témoigné une énorme croissance dans notre lectorat. Avec ces changements, nous avons collectivement décidé d'élargir l'équipe éditoriale de la RCESSC et d'embaucher un nouveau groupe de membres du personnel talentueux et passionnés qui ont déjà grandement contribué à la revue. Nous souhaitons la bienvenue à nos nouveaux rédacteurs adjoints de la RCESSC : Nick Athey, Erin Denton, Christina Deroche, Julie Hagan, et Dikla Yogev; et, à nos nouveaux réviseurs : Sanou Boroma, Jason Huang, Asheka Jackson, Eshan Jozaghi, Colin Scott et Kim Seida ! En plus d'une croissance du personnel, le site de la RCESSC a également subi quelques modifications pour correspondre à l'évolution et la croissance du lectorat. Notre nouveau site Web se trouve à www.cgjsc.ca. Ici, vous trouverez notre bloc-générique mis à jour avec des renseignements sur notre nouveau personnel, des liens vers les numéros précédents, des médias et des photos d'événements de la RCESSC mis à jour, ainsi que des liens vers le site du Système de journaux en ligne (OJS) de la RCESSC où les auteurs peuvent continuer à soumettre leurs oeuvres. Nous sommes également heureux d'annoncer que la RCESSC sera en partenariat avec le Réseau canadien de recherche sur le terrorisme, la sécurité et la société (TSAS), pour un numéro spécial à paraître à l'automne 2013. Dr Aurélie Campana, la Chaire de recherche du Canada sur le terrorisme et les conflits, a accepté de siéger en tant que rédacteur en chef invité et le TSAS a généreusement fait don de $500 \$$ à tous les auteurs des articles acceptés pour ce numéro.

Une fois par an, nous aimerions prendre le temps de remercier sincèrement le travail acharné et le dévouement de nos pairs examinateurs. Les bénévoles dévoués qui nous ont aidés à assurer la qualité de nos soumissions sont trop nombreux pour énumérer leurs noms ici. Cependant, nous vous invitons à consulter le bloc-générique du Journal (http://www.cgjsc.ca/masthead/peer-reviewers), où l'ensemble de nos examinateurs pour l'année écoulée ont été reconnus pour et leurs efforts et leur travail acharné.

Pour notre deuxième numéro, nous avons quatre articles très intéressants. L'oeuvre de Maureen Kihika, intitulé «Fantômes et ombres : Une histoire du racisme au Canada », élabore éloquemment l'argument selon lequel les effets du racisme institutionnalisé, qui ont duré plusieurs siècles, se font encore sentir par les Afro-Canadiens qui participent au marché du travail d'aujourd'hui. Suite à l'affirmation de Bakan que les pratiques racistes et hégémoniques sont fortement ancrés dans la structure politique du Canada, Kihika retrace chronologiquement les conséquences des politiques néo-libérales du début du 16ème siècle, en affirmant que ces politiques, sur la surface, semblent soutenir les idéaux du multiculturalisme et de l'inclusivité, mais en réalité, n'ont servi qu'à propager davantage les programmes capitalistes et impérialistes de ceux au pouvoir. En conséquence, il est mis en évidence à l'aide de statistiques officielles et de recherches antérieures, que les Afro-Canadiens continuent à lutter économiquement et sont positionnés dans l'échelon inférieur de la hiérarchie du marché du travail, comme le note Kihika. Avec peu de capacité de gain et peu de possibilités d'emploi stable, l'auteur suggère que ce groupe racialisé en particulier continue à subir les conséquences néfastes de structures anciennes d'oppression systémique.

La pièce de Nicole Etherington, «Le cheminement scolaire et de la santé au cours de la vie : un rôle pour l'Académie DSBN ? », met l'accent sur le lien constaté entre la stratification socio-économique et les inégalités de santé en examinant en particulier la mise en oeuvre de l'Académie du District School Board of Niagara (DSBN) dans la région de Niagara, en Ontario, Canada. Les recherches ont montré que si, en moyenne, une personne sur trois âgés de plus de vingt-quatre ans en Ontario ont une formation universitaire, dans la région de Niagara, le taux de l'éducation post-secondaire est beaucoup plus faible, à seule une 
personne sur cinq. Le District School Board of Niagara a créé l'Académie DSBN en 2011 pour répondre à ces faibles taux avec la prise de conscience que les personnes qui ne fréquentent pas ou n'obtiennent pas de diplôme d'une institution post-secondaire sont plus susceptibles d'éprouver à la fois une mauvaise santé physique et mentale plus tard dans la vie. Etherington aborde les objectifs attendus de l'Académie DSBN d'offrir des programmes sociaux et scolaires qui visent à encourager les étudiants à ne pas seulement obtenir un diplôme d'études secondaires, mais aussi à poursuivre avec succès une éducation post-secondaire. Avec une discussion approfondie des avantages et des désavantages cumulatifs qui se produisent dans les domaines de l'éducation et de la santé, l'auteur évalue l'impact de l'Académie DSBN et conclut que l'Académie a le potentiel d'offrir des ressources aux étudiants afin de trouver d'autres formes de capital comme moyen de contrecarrer les effets du capital initial sur la réussite scolaire, et donc les résultats de santé plus tard dans la vie.

L'oeuvre de Konstantin Petoukhov, "Transformer le legs des pensionnats indiens au Canada en affaire publique: une analyse critique de la sociologie publique de Michael Burawoy, » utilise de manière intéressante les quatre types de sociologie de Michael Burawoy pour encadrer le processus de transformation de «problèmes personnels » en « questions publique » dans le contexte des pensionnats indiens (IRS). Petoukhov commence en offrant une première discussion sur le fond et les résultats des IRS qui ont longtemps résonné dans les communautés autochtones, même si tous les IRS ont été officiellement fermés en 1996. Petoukhov concentre son analyse sur l'évaluation des quatre types de sociologie de Burawoy - politique, critique, professionnelle et publique - en ce qui concerne leur capacité à identifier les problèmes particuliers qui ont surgi de l'expérience des pensionnats indiens sur des questions d'intérêt public et des origines systémiques. L'auteur explore en détail chacune des quatre formes de Burawoy et conclut finalement qu'elles sont incompatibles en leur capacité à contribuer à une meilleure compréhension des problèmes privés créés par les IRS en tant que questions publiques.

La pièce d'Ehsan Jozaghi, «'La plus grande erreur que Dieu ait commise a été de créer les junkies' : pratiques d'injection non sécuritaires, discrimination des soins de santé et décès par surdose à Montréal, Canada » est centrée sur la question des équipements de drogue partagés par les utilisateurs de drogues injectables (UDI) à Montréal, Canada, qui a conduit à une augmentation de la prévalence du VIH et de l'hépatite C (VHC) depuis 2003. La recherche de Jozaghi est basée sur des récits qualitatifs réalisés par des entretiens semistructurés avec les UDI qui ont révélé leur implication dans les comportements à haut risque qui augmentent l'exposition au VIH, au VHC et à la transmission bactérienne à la fois pour les usagers de drogues et pour le grand public. L'auteur discute que certains des facteurs cumulatifs qui contribuent à l'augmentation de la morbidité et de la mortalité des UDI à Montréal, ainsi que le risque pour le grand public comprennent : l'utilisation de l'eau de flaque d'eau contaminée pour l'injection, la contrainte de devoir injecter dans des environnements insalubres comme les toilettes publiques ou les allées, le rejet des aiguilles usagées dans les espaces publics et les préjugés et le harcèlement par la police et le personnel de l'hôpital lorsqu'ils cherchent à obtenir un traitement. Jozaghi conclut avec la recommandation que Montréal bénéficierait grandement de la mise en oeuvre d'un centre d'injection supervisé, semblable à celle d'autres villes canadiennes comme Vancouver.

Encore une fois, nous tenons à adresser un grand merci à tous ceux et celles qui ont contribué à la réussite de la RCESSC, et à nos lecteurs et abonnés qui nous ont soutenus par l'entremise de lectorat et au sein des réseaux sociaux. Au nom de la RCESSC, profitez de ce deuxième numéro passionnant ! 